\title{
DENSE SUBGROUPS OF FINITE GROUPS
}

\author{
TERENCE M. GAGEN and PAUL M. WEICHSEL*
}

(Received 8 September 1969)

Communicated by G. E. Wall

\section{Introduction}

It is well known that in the category of all groups and homomorphisms, every epimorphism is onto. This result does not hold for certain other categories of groups. The condition that an epimorphism $\theta: G \rightarrow A$ is not onto is equivalent to the condition that $\theta(G)$ is a proper subgroup $A$ with the property that any two homomorphisms $\alpha, \beta$ on $A$ which agree elementwise on $\theta(G)$ must agree on A. Such a subgroup can be called dense (see e.g. [1]). Naturally the existence of such homomorphisms $\alpha$ and $\beta$ depend on the particular class of groups that is available. We will choose to work within the context of varieties even though many of the results will hold true for more modest classes of groups.

Definition. Let $H$ be a subgroup of the group $G$. Then $H$ is called dense in $G$ if for any pair of homomorphisms $\theta, \varphi: G \rightarrow K$ with $K$ a group in the variety generated by $G, \theta(h)=\varphi(h)$ for all $h \in H$ imples that $\theta=\varphi$. Otherwise $H$ is said to be not dense in $G$. (This is a variation of the notion of $X$-dense in [1] p. 327.)

In this paper we will study the existence of dense subgroups in finite groups. As we will shortly see, the notion of density is in a sense opposite from that of normality and so many of our results will involve subgroups of simple groups. In particular we will prove that the alternating group $A_{n}$ is dense in $A_{n+1}$ for $n \geqq 4$.

\section{Notation and preliminaries}

A variety generated by a group $G$ (class of groups $A_{\alpha}$ ) is the smallest class of groups containing $G$ (the class $A_{\alpha}$ ) and closed with respect to the operations of forming cartesian products, subgroups and factor groups. It is denoted by var $G$ $\left(\operatorname{var}\left\{A_{\alpha}\right\}\right)$. A variety can be characterized as that class of groups which satisfy a given set of identical relations, or laws. If $w$ is a set of words on some set of variables, then we denote by $w(G)$ the subgroup of the group $G$ generated by the

* Research supported by National Science Foundation Grant GP 7029. 
elements obtained by substituting all possible group elements for the variables in the words of $w . w(G)$ is called the verbal subgroup of G generated by $w$. A group is said to be critcal if the variety generated by the class of all proper subgroups and proper factor groups of $G$ does not contain $G$. All groups considered will be finite and we will use simple group to mean finite non-abelian simple group. We will use without proof the fact that finite simple groups are critical. (See, e.g. [2] p. 147).

First, a number of elementary results which follow directly from the definition.

Lemma 2.1. Let $N \triangleleft G$. Then $N$ is not dense in $G$.

Proof. Let $\theta$ be the natural map from $G$ to $G / N$ and $\varphi$ the trivial map from $G$ to $G / N$. Clearly $\theta$ and $\varphi$ agree on $N$ but not on $G$ and $G / N \in$ var $G$. (This proves that epimorphisms are onto in the category of all abelian groups.)

Lemma 2.2. Let $G=A B, A \triangleleft G$ and $A \cap B=1$. Then both $A$ and $B$ are not dense in $G$.

Proof. $A$ is not dense in $G$ from Lemma 2.1. Let $\theta, \varphi: G \rightarrow G$ be defined in $\theta(g)=g$ and $\varphi(g)=\varphi(a b)=b ; a \in A, b \in B$. Then $\theta$ and $\varphi$ agree on $B$ but clearly $\theta \neq \varphi$.

Lemma 2.3. If $A$ is dense in $G$ and $A \subseteq B$, then $B$ is dense in $G$.

Lemma 2.4. If $A$ is dense in $G$ and $N \subset A, N \geqslant G$, then $A / N$ is dense in $G / N$.

Proof. Let $\bar{\theta}, \bar{\varphi}: G / N \rightarrow K$ with $K \in \operatorname{var} G / N$ and $\bar{\theta}(a N)=\bar{\varphi}(a N)$ for all $a \in A$. Now define $\theta, \varphi: G \rightarrow K$ by $\theta(g)=\bar{\theta}(g N)$, and $\varphi(g)=\bar{\varphi}(g N)$. Since $K \in \operatorname{var} G / N, K \in \operatorname{var} G$. Now if $a \in A, \theta(a)=\bar{\theta}(a N)=\bar{\varphi}(a N)=\varphi(a)$. Thus $\theta$ and $\varphi$ agree on $A$. But $A$ is dense in $G$ and thus $\theta=\varphi$. Hence $\bar{\theta}=\vec{\varphi}$ and so $A / N$ is dense in $G / N$.

LemMa 2.5. Let $\theta$ be a nontrivial automorphism of a group $G$ which centralizes the subgroup $H$ of $G$. Then $H$ is not dense in $G$.

Proof. Let $\varphi$ be the identity map on $G$. Then $\theta \neq \varphi$ and so $H$ is not dense in $G$.

COROLlary 2.6. Let $H$ be a subgroup of $G$ with (centre of $H) \$$ (centre of $G$ ). Then $H$ is not dense in $G$.

Proof. Let $\theta$ be the inner automorphism induced in $G$ by a central element of $H$ which is not central in $G$ and apply Lemma 2.5.

\section{Dense subgroups of simple groups}

We have given a sufficient condition for a subgroup to be not dense in a group in Lemma 2.5. In this section we will show that if $G$ is a finite simple group, then this condition is also necessary. That is, we will prove: 
THEOREM 3.1. Let $H$ be a subgroup of the simple group $G$. Then $H$ is not dense in $G$ if and only if there exists a nontrivial automorphism of $G$ which centralizes $H$.

We first give a number of lemmas.

Lemma 3.2. (Kovács and Newman [3] Theorem 2). Let $N$ be a minimal normal subgroup of the group $K$ and suppose that var $K=\operatorname{var}(K / N)$. Then $K$ contains a nontrivial normal subgroup $M$ such that $N \cap M=1$.

Lemma 3.3. Let $G$ be a simple group and let $K \in$ var $G$. Let $N$ be a minimal normal subgroup of $K$ such that $K=G N, G \cap N=1$. Then $K=M \times N$ with $M \simeq G$.

Proof. Since $N$ is minimal normal in $K$ and $K / N \simeq G$, it follows from Lemma 3.2 that $K$ contains a normal subgroup $M$ such that $M \cap N=1$. Now $M N / N \exists K / N \simeq G$ which is simple and so $M N / N=K / N$. Thus $K=M N=$ $M \times N$. But $G \simeq K / N \simeq M$ and the lemma follows.

LeMmA 3.4. Let $G$ be a simple group and let $K$ be a group of minimal order satisfying the following properties

i) $K \in \operatorname{var} G$.

ii) $K$ is generated by the proper subgroups $G_{1}$ and $G_{2}$ with $G_{1} \simeq G_{2} \simeq G$.

iii) $G_{1} \cap G_{2} \supseteq H_{1} \neq 1$.

Then there exists a non-trivial automorphism of $G$ which centralizes $H, H$ being the image of $H_{1}$ under an isomorphism $G_{1} \simeq G$.

Proof. Since $K$ contains a subgroup isomorphic to $G$ and $K \in \operatorname{var} G, K$ is not critical and therefore $K$ is not simple. Let $N$ be a minimal normal subgroup of $K$. Since $G$ is simple $G_{1} \cap N=G_{2} \cap N=1$. For otherwise $N \supseteq G_{1}$ say, and since $G_{1} \cap G_{2} \neq 1, N \supseteq G_{2}$. But $N$ is a proper subgroup of $K$ contradicting the minimality of $K$. Now consider $K / N . G_{1} N / N$ and $G_{2} N / N$ generate $K / N$ and $G_{1} N / N \simeq G_{2} N / N \simeq G$. Further $\left[G_{1} N / N\right] \cap\left[G_{2} N / N\right] \supseteq H_{1} N / N \simeq H$. Thus by the minimality of $K$ we get $K=G_{1} N$ and by Lemma 3.3, $K=M \times N$ with $M \simeq G$.

Now suppose that $M=G_{1}$. Then since $G_{1} \otimes K, 1 \neq G_{1} \cap G_{2} \otimes G_{2}$ and hence $G_{1}=G_{2}$, a contradiction. Thus $G_{1} \neq M$ and similarly $G_{2} \neq M$. (We have already shown above that $G_{1} \cap N=G_{2} \cap N=1$.)

Now every element $g \in G_{1}$ is of the form $g=m_{1} n_{1}, m_{1} \in M, n_{1} \in N$ with $m_{1}=1$ if and only if $n_{1}=1$. For otherwise the set of elements of $G_{1}$ of the form $1 \cdot n$ or $m \cdot 1$ would form a proper normal subgroup of $G_{1}$. The projection map $\pi: G_{1} \rightarrow M$ is onto since $G$ is simple. Thus the mapping $\alpha: M \rightarrow N$ defined by $\alpha(m)=n$ if $m n \in G_{1}$ is an isomorphism into $N$ and hence the set of elements $\{n\}$ such that $m n \in G_{1}$ form a subgroup of $N$ isomorphic to $G$. Arguing the same way with $G_{2}$ it follows that $N$ contains a subgroup $\left\{n^{\prime}\right\}$ such that $m^{\prime} n^{\prime} \in G_{2}$ and that $\left\{n^{\prime}\right\} \simeq G$. Now suppose that $\{n\} \neq\left\{n^{\prime}\right\}$. Thus since $G_{1} \cap G_{2} \neq 1,\{n\} \cap\left\{n^{\prime}\right\} \neq 1$ 
and $N$ satisfies hypotheses (i), (ii), and (iii). But $K$ is of minimal order, and hence $N \simeq G$.

We now define the mapping $\alpha: G_{1} \rightarrow G_{2}$ as follows $\alpha(m n)=m n^{\prime}$ for all $m n \in G_{1}$. This mapping is well-defined and onto $G_{2}$. It is clearly a homomorphism and in fact an isomorphism. Now since $G_{1} \neq G_{2}$, the mapping $\alpha^{\prime}: N \rightarrow N$ defined by $\alpha^{\prime}(n)=n^{\prime}$ when $m n \in G_{1}$ and $\alpha(m n)=m n^{\prime}$ is a nontrivial automorphism of $N$. Further $\alpha^{\prime}$ centralizes $H_{1}^{\prime} \simeq H_{1}$ in $N$ since $H_{1} \in G_{1} \cap G_{2}$. Thus there is a nontrivial automorphism of $G$ which centralizes $H$.

Proof OF TheOrem 3.1. If there exists a nontrivial automorphism of $G$ which centralizes $H$, then by Lemma $2.5, H$ is not dense in $G$.

Suppose that $H$ is not dense in $G$. Then there exists a group $K \in \operatorname{var} G$ and a pair of homomorphisms $\theta, \phi: G \rightarrow K$ such that $\theta(h)=\phi(h)$ for all $h \in H$ and there exists $g \in G$ such that $\theta(g) \neq \phi(g)$. We may choose $K$ to be of minimal order subject to these conditions and it then follows that $K$ is generated by $\theta(G)$ and $\phi(G)$. Clearly $\theta(G) \cong \phi(G) \cong G$ since $G$ is simple and $\theta, \phi$ are both non-trivial. Now suppose that $\theta(G)=\phi(G)=K$. Then the mapping $\alpha: K \rightarrow K$ defined by $\alpha \theta(g)=\phi(g)$ is clearly a nontrivial automorphism of $G$ which fixes $H$. Thus we assume that $\theta(G)$ and $\phi(G)$ are proper subgroups of $K$. Further since $G$ is simple and neither $\theta$ nor $\phi$ is the trivial map $G \simeq \theta(G) \simeq \phi(G)$. Hence all of the conditions of Lemma 3.4 are satisfied and the theorem is proven.

Corollary 3.5. $A_{n}$ is dense in $A_{n+1}$ for $n \geqq 4$.

Proof. We first remark that $A_{n}$ is thought of as a subgroup of $A_{n+1}$ in the obvious way. By virtue of Theorem 3.1. we need only verify that no automorphism of $A_{n+1}$ centralizes $A_{n}$ except the identity automorphism. Now the automorphism group of $A_{n+1}$ if $S_{n+1}$ unless $n=5$, and clearly conjugation by an element of $S_{n+1}$ will centralize $A_{n}$ only if it is the identity permutation. If $n=5$, the automorphism group $T$ of $A_{6}$ contains $S_{6}$ with index 2. Any element of $T$ not in $S_{6}$ interchanges the two classes of groups isomorphic to $A_{5}$ and so certainly does not centralize any one of them.

\section{Density in finite groups}

P. M. Neumann has proven that no proper subgroup of a finite soluble group is dense ([1] P. 328. It is easy to generalize the result as follows.

THEOREM 4.1. Let $N \triangleleft G$ such that $G / N$ is soluble. Then if $H$ is a subgroup of $G$ containing $N, H$ is not dense in $G$.

Proof. By the result cited above, $H / N$ is not dense in $G / N$. Thus by Lemma $2.4 H$ is not dense in $G$.

We are thus led to ask whether analagous results may be obtained for groups, 
each of whose maximal normal subgroups have simple factors. We will prove a very special theorem along these lines and then show by example that the more general statement is in fact false.

Theorem 4.2. Let $N \notin G$ such that $G / N$ is simple and $N$ is central in $G$. Let $H$ be a proper subgroup of $G$ containing $N$. Then $H$ is dense in $G$ if and only if $H / N$ is dense in $G / N$.

Proof. If $H$ is dense in $G$, then $H / N$ is dense in $G / N$ by Lemma 2.4.

Conversely assume that $H$ is not dense in $G$, but $H / N$ is dense in $G / N$. Then there is a group $K$ in $\operatorname{var} G$ and maps $\theta, \phi: G \rightarrow K$ such that $\theta(h)=\phi(h)$ for all $h \in H$ but $\theta(g) \neq \phi(g)$ for at least one $g \in G$. As in the proof of Theorem 3.1 we choose $K$ so that $K$ is generated by $\theta(G)$ and $\phi(G)$.

We have $\operatorname{ker} \theta=\operatorname{ker} \phi \subseteq N$. For if $\operatorname{ker} \theta, \operatorname{ker} \phi \subseteq H$, obviously kel $\theta=$ ker $\phi \subseteq N$ since $G / N$ is simple. If say $\operatorname{ker} \theta \ddagger H, N \subset N \operatorname{ker} \theta \unlhd G$ and so $G=N$ ker $\theta$. Then for all $h \in H$

$$
\begin{aligned}
\phi(h)=\theta(h) & =\theta(n k) \text { where } n \in N, k \in \operatorname{ker} \theta \\
& =\theta(n)=\phi(n) .
\end{aligned}
$$

Thus $h n^{-1} \in \operatorname{ker} \phi$ and so

$$
H \subseteq N \operatorname{ker} \phi \boxminus G .
$$

Therefore $G=N \operatorname{ker} \theta=N \operatorname{ker} \phi$. Since $N$ is abelian, $\theta(G)$ and $\phi(G)$ are both abelian. Then for all $g \in G, h \in H$

$$
\begin{aligned}
\theta\left(g^{-1} h g\right) & =\theta(g)^{-1} \theta(h) \theta(g) \\
& =\theta(h) \quad \text { since } \theta(G) \text { is abelian } \\
& =\phi(h)=\phi\left(g^{-1} h g\right) .
\end{aligned}
$$

Hence $\theta$ agrees with $\phi$ on all conjugates $H^{g}$ of $H$. But $G=\left\langle H^{g}, N: g \in G\right\rangle$ and so $\theta=\phi$ on $G$. This is a contradiction.

Now suppose that $\theta(G)=\phi(G)=K$. Then consider the map $\alpha: K \rightarrow K$ defined by $\alpha \theta(g)=\phi(g)$. Since $\operatorname{ker} \theta=\operatorname{ker} \phi, \alpha$ is an automorphism of $K$ which centralizes $\theta(H)$. Now let $\alpha^{\prime}: K / \theta(N) \rightarrow K / \theta(N)$ be defined by $\alpha^{\prime}\{k \theta(N)\}=$ $\alpha(k) \theta(N)$. Then $\alpha^{\prime}$ centralizes $\theta(H) / \theta(N)$ and hence $\alpha^{\prime}$ is the identity since $K / \theta(N) \simeq G / N$ and $H / N$ is dense in $G / N$. Thus $\alpha$ is a nonidentity automorphism of $K$ which is the identity on $\theta(H)$ and whose induced map $\alpha^{\prime}$ is the identity on $K / \theta(N)$. Consider an arbitrary conjugate of an element of $\theta(H)$, say $\theta(g)^{-1} \theta(h) \theta(g)$ $=\theta\left(g^{-1} h g\right)$. Since $\alpha^{\prime}$ is the identity on $K / \theta(N), \alpha \theta(g)=\theta(g) \theta(n), n \in N$.

Thus $\alpha \theta\left(g^{-1} h g\right)=\alpha \theta(g)^{-1} \alpha \theta(h) \alpha \theta(g)=(\theta(g n))^{-1} \theta(h)(\theta(g n))=\theta(n)^{-1}$ $\theta(g)^{-1} \theta(h) \theta(g) \theta(n)$. But $n$ is central in $G$ and hence $\theta(n)$ is central in $K$. Thus $\alpha \theta\left(g^{-1} h g\right)=\theta\left(g^{-1} h g\right)$. But the set $\left\{g^{-1} h g \mid g \in G\right.$ and $\left.h \in H\right\}$ generates $G$ and hence the set of fixed elements of $\alpha$ generates $K$, a contradiction. Therefore $\theta(G) \neq \varphi(G)$. 
Now let $w$ be the set of words which characterizes $\operatorname{var}(G / N)$, and let $\theta^{*}$, $\varphi^{*}: G / N \rightarrow K / w(K)$ be defined by $\theta^{*}(g N)=\theta(g) w(K), \varphi^{*}(g N)=\varphi(g) w(K)$. Clearly $K / w(K) \in \operatorname{var}(G / N)$ and $\theta^{*}$ agrees with $\varphi^{*}$ on $H / N$. The proof will be complete if we can show that $\theta^{*}(g N) \neq \varphi^{*}(g N)$ for some $g N \in G / N$.

Thus assume that $\theta^{*}(g N)=\varphi^{*}(g N)$ for all $g N \in G / N$. Now $K / w(K)$ is clearly generated by $\theta^{*}(G / N)$ and $\varphi^{*}(G / N)$. Thus $K w(K)=\theta^{*}(G / N)=\varphi^{*}(G / N)$ and hence $K$ is generated by its subgroups $\theta(G)$ and $w(K)$. We may without loss of generality assume that $\operatorname{ker} \theta=\operatorname{ker} \varphi=N$. For otherwise we may replace $\theta$ and $\varphi$ by $\theta^{\prime}=\alpha \theta$ and $\varphi^{\prime}=\alpha \varphi$ where $\alpha: K \rightarrow K / \theta(N)$ is the natural map. Clearly $\theta^{\prime}(h)=\varphi^{\prime}(h)$ for all $h \in H$ and there is at least one $g \in G$ such that $\theta^{\prime}(g)=$ $\theta(g) \theta(N) \neq \varphi(g) \varphi(N)=\varphi^{\prime}(g)$. Thus assume $\operatorname{ker} \theta=\operatorname{ker} \varphi=N . K=\theta(G) w(K)$ $=\varphi(G) w(K)$. But since $w(G)$ is central in $G, w(A)$ is central in $A$ for every group $A \in \operatorname{var} G$, and hence $w(K)$ is central in $K$. Hence since $\theta(G)$ is simple $K=\theta(G) \times$ $w(K)$. But $\varphi(G)$ is a subgroup of $K$ and is isomorphic to $\theta(G)$. However $w(K)$ is abslian and hence $\varphi(G)=\varphi(G)$, a contradiction. Hence $H / N$ is not dense in $G / N$ and the theorem follows.

\section{An example}

The following group $G$ is an example of a group with the property that $G / N$ contains a dense proper subgroup $H / N$ for some normal subgroup $N$ but $H$ is not dense in $G$. This shows that the condition that $N$ is central in $G$ cannot be removed in Theorem 4.3.

Consider the group $N_{1}=\left\langle a_{1}, a_{2}, a_{3}, a_{4}, a_{5}\right\rangle$ where $a_{i}^{2}=1,\left[a_{i}, a_{j}\right]=1$ for all $i, j=1,2, \cdots, 5$. Now let $A \cong A_{5}$ and let $G_{1}=A N_{1}$ where $N_{1} \otimes G_{1}$ and $A$ acts on $N_{1}$ in the obvious way. Clearly $N_{1}=N \oplus\left\langle a_{1}+a_{2}+a_{3}+a_{4}+a_{5}\right\rangle$ where $N=\left\langle a_{1}+a_{2}, a_{1}+a_{3}, a_{1}+a_{4}, a_{1}+a_{5}\right\rangle$ and $N$ is an $A$-module. Let $G=N A$.

Now if $N H / N \subseteq G / N$ is isomorphic to $A_{4}$ then by Corollary 3.5, NH/N is dense in $G / N$. Suppose that $H \subseteq A$ acts on the symbols $1,2,3,4$. Then consider $x=a_{1}+a_{2}+a_{3}+a_{4} \in N$. Clearly the inner automorphism $\alpha$ of $G$ induced by $x$ centralizes $N$ and $H$. However $\alpha$ is not the identity on $G$ and so $N H$ is not dense in $G$.

\section{References}

[1] P. M. Neumann, 'Splitting groups and projectives in varieties of groups', Quart. J. of Math. (Oxford Ser.) 18 (1967), 325-332.

[2] Hanna, Neumann, Varieties of Groups (Ergebnisse der Mathematik und ihrer Grenzgebiete, Band 37, Springer-Verlag, Berlin, 1967).

[3] L. G. Kovécs and M. F. Newman, 'Minimal verbal subgroups', Proc. Cambridge Philos. Soc. 62 (1966), 347-350.

University of Sydney, Sydney, Australia

University of Illinois, Urbana, Illinois, U.S.A. 